[5] V. C. Pezoulas, et al., "Towards the establishment of a biomedical ontology for the primary Sjögren's Syndrome," in IEEE Eng. Med. Biol. Soc., 2018;4089-92.

[6] K. Kourou, et al., "Cohort Harmonization and Integrative Analysis from a Biomedical Engineering Perspective," IEEE Rev. Biomed. Eng., 2018.

Acknowledgement: * This project has received funding from the European Union's Horizon 2020 research and innovation programme under grant agreement No 731944 and from the Swiss State Secretariat for Education, Research and Innovation SERI under grant agreement 16.0210.

Disclosure of Interests: Vasileios Pezoulas: None declared, Themis Exarchos: None declared, Aliki Venetsanopoulou: None declared, Evangelia Zampeli Speakers bureau: Roshe, Astrazeneca, Saviana Gandolfo: None declared, Salvatore De Vita Grant/research support from: Roche, Pfizer, Abbvie, Novartis, BMS, MSD, Celgene, Janssen, Consultant for: Roche, Foteini N. Skopouli: None declared, Athanasios Tzioufas Grant/research support from: ABBVIE, PFIZER, AMGEN, NOVARTIS, GSK, Dimitrios Fotiadis: None declared DOI: 10.1136/annrheumdis-2019-eular.4139

\section{AB0167 1 SINGLE CELL RNA EXPRESSION IN LUPUS NEPHRITIS COMPARING AFRICAN-AMERICAN AND CAUCASIAN PATIENTS IDENTIFIES DIFFERENTIAL EXPRESSION OF TYPE I INTERFERON PATHWAY}

Andrea Fava ${ }^{1}$, Yuji Zhang ${ }^{2}$, Nir Hacohen ${ }^{3}$, Arnon Arazi ${ }^{4}$, Celine Berthier ${ }^{5}$, Deepak Rao ${ }^{3,6}$, Michael Brenner ${ }^{3,6}$, David Wofsy ${ }^{7}$, Anne Davidson $^{8}$, Matthias Kretzler ${ }^{9}$, David Hildeman ${ }^{10}$, E. Steve Woodle ${ }^{10}$, Betty Diamond ${ }^{8}$, Michelle A. Petri ${ }^{1}$, on behalf of Accelerating Medicine Partnership. ${ }^{1}$ Johns Hopkins University, Baltimore, United States of America; ${ }^{2}$ University of Maryland, Biostatistics, Baltimore, United States of America; ${ }^{3}$ Harvard Medical School, Boston, United States of America; ${ }^{4}$ Broad Institute, Cambridge, United States of America; ${ }^{5}$ University of Michigan, Nephrology, Ann Arbor, United States of America; ${ }^{6}$ Brigham and Women's Hospital, Boston, United States of America; ${ }^{7}$ UCSF Medical Center, San Francisco, United States of America; ${ }^{8}$ The Feinstein Institute for Medical Research, Manhasset, United States of America; ${ }^{9}$ University of Michigan, Ann Arbor, United States of America; ${ }^{10}$ University of Cincinnati, Cincinnati, United States of America

Background: African-American ethnicity is associated with a 3-fold higher risk of developing systemic lupus erythematosus (SLE). In addition, there is an increased risk of lupus nephritis (2-fold), high-risk histological features, and resistance to treatment. This may account for the increased mortality rate compared to Caucasian patients, especially in women.

Objectives: In Phase One of the Accelerating Medicines Partnership (AMP) study, we used single-cell RNA sequencing on kidney biopsies from patients with active lupus nephritis to identify pathways that were differentially expressed in African-American patients.

Methods: Single cell RNA sequencing was performed on renal biopsies obtained for clinical purpose for active nephritis using CEL-Seq2. Cell clusters with similar expression profile were identified using t-distributed stochastic neighbor embedding (t-SNE). First, the relative abundance of a cluster in AAs compared to Caucasian was determined using a logistic mixed model. Second, the differential expression profile was determined for each cell cluster and we applied Ingenuity Pathway Analysis (IPA) (QIAGEN Bioinformatics) to identify pathways of interest. Results: Samples from 13 AA and 7 Caucasian patients were obtained. Of the 3097 sequenced cell libraries, we used 2354 which passed our quality filter for a total of 30155 unique molecular identifiers. We identified 16 cell clusters including CD4, CD8, B and plasma cells, NK, myeloid cells, and tubular cells. We identified 2 cell clusters unique to African-American patients, a $T$ and a B cell population with high expression of interferon inducible genes. We also identified that same cell populations may have differential gene expression profiles across ethnicity. For example, CD4 T cells in African-Americans have a higher expression of type 1 and type 2 interferon pathways. In contrast, myeloid cells have several upregulated pathways in Caucasians, including ERK/MAPK signaling.

Conclusion: African-American lupus nephritis patients may have a stronger interferon pathway activation in infiltrating immune cells. Several other pathways, including ERK/MAPK, are differentially expressed in infiltrating cells based on ethnicity. These results suggest that ethnicity might predict a response to both current and upcoming treatments, paving the way for a more personalized approach to treatment in lupus nephritis. Further work in Phase 2 of AMP will confirm and extend these findings.

Disclosure of Interests: Andrea Fava: None declared, Yuji Zhang: None declared, Nir Hacohen: None declared, Arnon Arazi: None declared, Celine Berthier: None declared, Deepak Rao: None declared, Michael Brenner Grant/research support from: Roche: sponsored research agreement on stromal cells (but has nothing to do with checkpoint related disease), Consultant for: GSK: consultant. (I am part of their immunology network, a group of about 8 immunologists who advise them regularly and broadly in the areas of inflammation and infection)., David Wofsy Consultant for: GlaxoSmithKline - Member, data safety monitoring board

Novartis - Member, data safety monitoring board

Celgene - member, scientific advisory board, Anne Davidson: None declared, Matthias Kretzler: None declared, David Hildeman: None declared, E. Steve Woodle: None declared, Betty Diamond: None declared, Michelle A Petri Share holder of: Pfizer, Merck, Grant/research support from: AstraZeneca, Exagen, Consultant for: Eli Lilly, GSK, Merck EMD Serono, Janssen, Amgen, Novartis,

Quintiles, Exagen, Inova Diagnostics, AstraZeneca, Blackrock,

Glenmark, UCB, and the Annenberg Center for Health Sciences DOI: 10.1136/annrheumdis-2019-eular.5673

\section{$\mathrm{AB} 0168$ \\ 1,25 DIHYDROXYVITAMIN D IMPROVEMENTS ARTHRITIS BUT DOES NOT ALLEVIATE RENAL DAMAGE IN PRISTANE-INDUCED LUPUS MODEL}

Eduarda Freitas $^{1,2}$, Thaís Karnopp ${ }^{1,2}$, Jordana Miranda de Souza Silva ${ }^{1,2}$, Rafaela Cavalheiro Do Espírito Santo ${ }^{1,2}$, Thales Hein Da Rosa ${ }^{1,2}$, Mayara Souza de Oliveira ${ }^{1}$, Fabiany Gonçalves ${ }^{2}$, Francine Hehn de Oliveira ${ }^{3}$, Pedro Guilherme Schaefer ${ }^{3}$, Odirlei Monticielo ${ }^{1,2}$. ${ }^{1}$ Universidade Federal do Rio Grande do Sul, Porto Alegre, Brazil; ${ }^{2}$ Laboratório de Doenças Autoimunes, Porto Alegre, Brazil; ${ }^{3}$ Hospital de Clínicas de Porto Alegre, Porto Alegre, Brazil

Background: SLE is an autoimmune disease, characterized by the production of autoantibodies and tissue damage. The role of vitamin D (VD) in SLE has been increasingly explored. VD is a steroid hormone notably involved in the regulation of calcium and bone homeostasis, and its deficiency has been implicated in the development of SLE and other connective tissue diseases $(2,3)$. The study of different murine models has provided a better understanding of these autoimmune phenomena. Pristane-induced lupus (PIL) represents a suitable model to study factors that could influence the induction and/or progression of SLE, including genetic factors.

Objectives: To evaluate the development and evolution of SLE after 1,25(OH)2D supplementation in the PIL model.

Methods: Female BALB/c mice divided into 3 groups: $C O, P I L$ and PIL+VD. Lupus was induced in PIL and VD groups using pristane. VD group received a subcutaneous injection of calcitriol [2ug/kg] in PBS-Tween 20 buffer every second day during 180 days. Body weight, arthritis clinical score, edema and articula nociception was measured. On day 150 after pristane induction, the animals were placed in individual metabolic cages for urine collection for a period of $12 \mathrm{~h}$. Protein levels on urine were analyzed using urine test strips. At the end of the experimental period, serum, tibiotarsal joint and kidneys was collected. Hind paws were collected to confirm the development of arthritis by histological analysis with HE staining. The glomerular cellularity was quantified by counting the total cell nuclei per glomerulus on HE slides. Immune complex deposition ( $\lg \mathrm{G}$ and $\operatorname{lgM}$ ) in kidney was examined by direct immunofluorescence. IL-2, IL-4, IL-6, IFN- $\gamma$ and TNF- $\alpha$ were measured by Luminex technology in serum. Data was analyzed with ANOVA Two-Way followed by Bonferroni and independent sample t-test. $p<0.05$ was considered significant. All data are represented as mean \pm SD.

Results: PIL group showed arthritis and kidney injury, characterized by increased proteinuria, glomerular mesangial expansion and inflammation. Moreover, PIL model showed increased levels of IL-6, TNF- $\alpha$ and IFN- $\gamma$ in serum. VD treatment reduced arthritis incidence compared $\mathrm{PIL}(42 \mathrm{vs} 85 \% ; \mathrm{p}<0.01)$ at the end of the experimental period. The arthritis clinical score $(1.00 \pm 1.15 v s 2.85 \pm 1.34 ; p<0.001)$ and the hind paws edema $(0.20 \pm 0.03 v s 0.24 \pm 0.05 \mathrm{~mL} ; \mathrm{p}<0.05)$ in the VD group were also attenuated in relation to the PIL at day 180 after induction. VD was able to reduce synovial hyperplasia $(0(0,1) v s 2(2,3) ; \mathrm{p}<0.05)$, erosion in bone $(0(0,1)$ vs2(2,2);p<0.05) and cartilage $(1(0,2) v s 3(2,3) ; p<0.05)$ when compared to the PIL group. Treatment with VD was not able to reduce proteinuria levels $(44.28 \mathrm{vs} 47.14$ $\mathrm{mg} / \mathrm{dL})$, decrease mesangial hypercellularity (31.48 $\pm 2.6 \mathrm{vs} 33.12 \pm 3.4$ nucleo/glo meruli) or IgG (17.88 $\pm 6.0 v s 24.04 \pm 6.2)$ and $\lg M(12.62 \pm 6.9 v s 15.57 \pm 3.7)$ deposition in the kidney. VD supplementation did not alter IL-6, TNF- $\alpha, \mathrm{IL}-2$ and IL-4 cytokine levels, but reduce IFN- $y$ levels $(p<0.01)$

Conclusion: VD improves arthritis but does not influence renal injury despite reducing IFN-y levels. These results support that the role of VD may be different depending on acting site, what could explain different responses according clinical phenotype. Therefore, further investigations of VD are needed to explore the supplement dosage, timing, and the molecular basis in SLE.

\section{REFERENCES}

[1] Pons-Estel GJ, et al. Seminars in Arthritis and Rheumatism. 2010. 2 Kamen DL, et al. Autoimmun Rev 2006; 5: 114-117. 3 Orbach H, et al. Ann N Y Acad Sci 2007; 1109: 385-400.

Acknowledgement: FIPE/HCPA, CAPES, CNPq Universal

Disclosure of Interests: None declared

DOI: 10.1136/annrheumdis-2019-eular.6328 